\section{MS45-P4 On the application of leverage analysis to parameter precision using area detector strategies}

Pascal Parois ${ }^{1}$, Richard I. Cooper ${ }^{1}$

1. University of Oxford, Chemistry research laboratory, 12 Mansfield road, Oxford, UK

email: pascal.parois@chem.ox.ac.uk

Analysis of the infuence of measurements on an existing crystallographic model allows the identification of the most useful additional data to measure to improve the precision of specific model parameters. We show that as a result of the distribution of those diffraction measurements in reciprocal space, the technique is more appropriate for certain parameters and derived functions when using an area detector. Remeasurement of specific diffraction peaks from single crystals in order improve the precision of specific parameters is considered in the context of data collection strategy using an area detector. By considering strategies for recollecting small sets of data, we show that certain parameters are more amenable to being optimized in this manner.

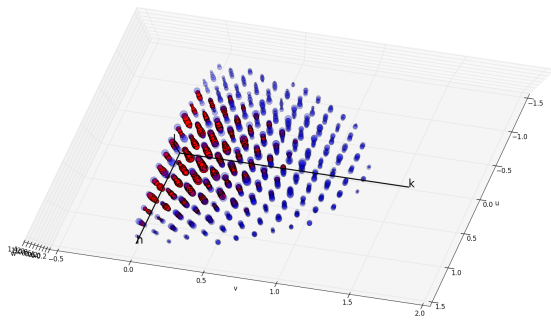

Figure 1. Estimated improvement in variance $\Delta \mathrm{V}$ of all the reflections $(\mathrm{h}, \mathrm{k}, \mathrm{l})$ in real space $(\mathrm{u}, \mathrm{v}, \mathrm{w})$. The area of the discs is proportional to $\Delta \mathrm{V}$ on a non linear scale. The red reflections are part of an optimised short data collection strategy calculated by our software to optimise the variance of a $\mathrm{C}-\mathrm{H}$ bond.

Keywords: Error analysis, Diffraction experiment strategy, Area detectors

\section{MS45-P5 XtaLAB Synergy: Fast, Precise, Intelligent.}

Fraser J. White ${ }^{1}$, Alexandra Griffin ${ }^{1}$, Dyanne Cruickshank ${ }^{1}$, Mathias Meyer $^{2}$, Damian Kucharczyk ${ }^{2}$

\section{Rigaku Oxford Diffraction, Sevenoaks, UK}

2. Rigaku Oxford Diffraction, Wrocław, Poland

email: fraser.white@rigaku.com

Performing data collections which are not only high quality but also efficient is a key requirement of diffractometer users around the world, either to enable high throughput, or simply maximise data quality in a given time. The new XtaLAB Synergy represents improvements in both hardware and software design. Advancements have been made not only in the microfocus sources but also in the goniometer hardware. The sample environment has been completely redesigned allowing ample space for easier access or incorporation of specialist equipment. The combination of these enhancements leads to better data quality in shorter data collection times for both weakly and strongly diffracting crystals.Ensuring the best possible use of the diffractometer hardware and the data it provides requires high performance software. The CrysAlisPro software package is under continual development in order to provide enhanced automation features, new options for sample screening, data collection and improvements to data processing algorithms. We compares the previous generation of Rigaku Oxford Diffraction diffractometer technology to the XtaLAB Synergy.

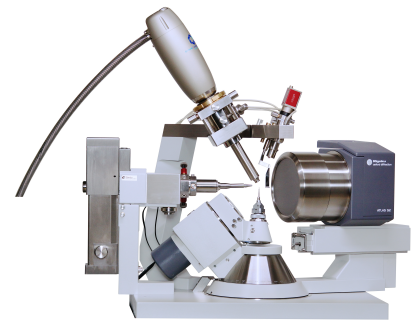

Figure 1. The XtaLAB Synergy with Atlas S2 detector

Keywords: hardware,diffractometer,high-speed,efficient 